\title{
Trivium
}

Revue franco-allemande de sciences humaines et sociales - Deutsch-französische Zeitschrift für Geistesund Sozialwissenschaften

1 | 2008

"Iconic Turn » et réflexion sociétale

\section{« Iconic Turn » et réflexion sociétale}

Introduction

\section{Bernd Stiegler}

Traducteur : Denis Trierweiler

\section{OpenEdition}

\section{Journals}

Édition électronique

URL : http://journals.openedition.org/trivium/308

DOI : $10.4000 /$ trivium.308

ISSN : 1963-1820

Éditeur

Les éditions de la Maison des sciences de l'Homme

Référence électronique

Bernd Stiegler, « «I Iconic Turn » et réflexion sociétale », Trivium [En ligne], 1 | 2008, mis en ligne le 08 avril 2008, consulté le 22 septembre 2020. URL : http://journals.openedition.org/trivium/308 ; DOI : https://doi.org/10.4000/trivium.308

Ce document a été généré automatiquement le 22 septembre 2020.

\section{c) (ㅇ) $\ominus$}

Les contenus des la revue Trivium sont mis à disposition selon les termes de la Licence Creative Commons Attribution - Pas d'Utilisation Commerciale - Pas de Modification 4.0 International. 


\title{
«Iconic Turn » et réflexion sociétale
}

\author{
Introduction \\ Bernd Stiegler \\ Traduction : Denis Trierweiler
}

1 Il est rare que le titre d'un livre devienne le nom d'un programme théorique. Mais, s'agissant du livre publié par Richard Rorty, The Linguistic Turn, c'est incontestablement le cas. ${ }^{1}$ Rorty a réuni les débats philosophiques de l'époque sous un concept prégnant, qui associait divers courants et, simultanément, établissait une position théorique programmatique. Celle-ci - selon Rorty - se concevait en se démarquant d'un modèle dominant toute la tradition philosophique, qui misait sur la visualité dans la théorie de la connaissance, et qui, non seulement déterminait la réflexion philosophique, mais, ainsi qu'il l'écrit, la maintenait prisonnière. La concentration sur le langage devait libérer la philosophie de son emprisonnement dans l'image et la visualité. Dans la publication de l'anthologie qu'il dirigea, Rorty entreprit des tentatives, encore plutôt tâtonnantes, pour sonder les possibles conséquences de cette nouvelle orientation de la philosophie. Puis, dans son œuvre majeure, L'homme spéculaire, il entreprit une critique décidée de la philosophie comme critique de l'image. "If the traditional "spectorial" account of knowledge is overthrown ", écrivit Rorty dans sa préface à The Linguistic Turn, «the account of knowledge which replaces it will lead to reformulations everywhere else in philosophy, particularly in metaphilosophy. $»^{2}$ Dans L'homme spéculaire, il se livre plus précisément à une critique des images et des métaphores, à une critique philosophique de cette «histoire de la domination des métaphores visuelles $» .^{3}$

«Ce ne sont pas des propositions, mais des images, peut-on lire là, non pas des déclarations, mais des métaphores qui dominent la plus grande part de nos convictions philosophiques. L'image, qui enferme la philosophie traditionnelle, est

l'image de la conscience comme d'un grand miroir. $»^{4}$

Et c'est précisément ce qu'il importe de questionner, de critiquer, de déconstruire.

2 La frappe, par Rorty, du concept de Linguistic Turn, s'est rapidement étendue, et bien au-delà de son programme philosophique. Un paradigme langagier est devenu constitutif de domaines aussi divers que la philosophie analytique, la sémiotique et la sémiologie, le structuralisme et le poststructuralisme (ou néo-structuralisme), ou 
encore l'herméneutique. "Le monde comme texte» a été érigé en une nouvelle métaphore, qui n'a pas tardé à devenir aussi dominante que la métaphore spéculaire de la tradition philosophique critiquée par Rorty. Dans son livre Downcast Eyes, Martin Jay constatait une véritable fixation langagière de la pensée, qui serait, de son point de vue, caractéristique de la philosophie du $\mathrm{xx}^{\mathrm{e}}$ siècle, et qui se distinguerait par un effacement conséquent de la visualité et de représentativité : iconoclasme et iconophobie sont devenus un programme philosophique, et même la critique derridienne du logocentrisme n'a pas permis de sortir du cercle langagier clos. Cependant, cette domination du paradigme langagier, qui n'est pas entièrement dénuée d'ambivalence chez Rorty, n'allait pas rester sans contradicteurs. La visualité et les images firent retour, et avec force. Dans les années 1990, W.J.T. Mitchell et Gottfried Boehm exigèrent presque simultanément des deux côtés de l'Atlantique une nouvelle orientation, un Turn supplémentaire de la vis théorique ${ }^{5}$, tournant qui serait requis entre autres parce que la signification sociale et l'omniprésence des images seraient devenues incommensurables, tandis que la théorie ne serait en aucune façon à la hauteur de l'événement. Il y est question de déluges d'images, de «l'ordre immanent et de la réflexivité des images $\aleph^{6}$, de cette situation étrangement paradoxale que les images auraient acquis un pouvoir immense, tout en provoquant simultanément une véritable angoisse: que la réalité ne se trouve entièrement sous la domination des images. Puis, cette nouvelle confrontation théorique avec les images, la visualité et la représentativité s'appuie également sur l'observation, aussi sobre que pertinente, selon laquelle les images jouent déjà un rôle central dans de nombreux domaines scientifiques, alors qu'elles n'entrent que peu à peu dans le champ de vision de l'attention théorique. ${ }^{7}$ Dans la pratique de la science a lieu depuis longtemps ce qui n'a encore qu'à peine dépassé le seuil de l'attention dans la théorie.

En $1992^{8}$, en opposition tranchée à l'iconoclasme et à l'iconophobie du Linguistic Turn', W. J. T. Mitchell revendique un Pictorial Turn qui proclame une capacité théorique affirmée des images qui se démarque de l'histoire de l'art traditionnelle d'une part, et d'une analyse des relations texte-image dominée par le langage d'autre part, et cherche à établir avec elles une véritable confrontation théorique. Ce qui était en jeu pour Mitchell était, ainsi que le formule Willibald Sauerländer, « un concept pour le nouveau rôle des images dans la communication sociale ${ }^{10}$ Dans l'espace germanophone original, ce fut Gottfried Boehm qui, en 1994, exigea de manière tout aussi programmatique un iconic turn dans son livre Was ist ein Bild ? [Qu'est-ce qu'une image] ${ }^{11}$ ? Tandis que Mitchell et de nombreux autres théoriciens dans l'espace anglophone concédaient au visuel surtout une force subversive et critique envers l'idéologie, en d'autres termes une force résolument sociale et politique, qui reçut d'importantes impulsions des débats des Cultural Studies - ce qui ouvrit à leur tour ceux des Visual Studies, des Cultural Studies et des Visual Culture Studies (cette triade étant symptomatique pour l'étrange situation d'un mélange théorique qui a toujours compris aussi le Pictorial Turn comme un Cultural Turn) -, Boehm se concentra, quant à lui, sur une expressivité et logique propres de l'image, recourant explicitement à la tradition herméneutique et transposant aux images la phrase de Gadamer : «L'être qui peut être compris est langage. » Ce faisant, Boehm comprend l'Iconic Turn comme la continuation logique du Linguistic Turn, comme une tentative pour comprendre "l'image en tant que logos ", comme un acte fondateur de sens. ${ }^{12}$ 
Ce qui est commun aux deux positions est la tentative de saisir par le concept un langage propre aux images. Les deux transgressent les limites de la science de l'art classique. Les deux entreprennent une critique décidée du logocentrisme de la théorie (philosophique, mais pas uniquement philosophique). Les deux tentent de mettre au jour la représentativité en tant que principe inhérent au langage. En revanche, les deux se séparent sur la question de savoir dans quelle mesure l'Iconic Turn ou le Pictorial Turn devrait nécessairement inclure aussi une réflexion sociale. Cependant tous deux ont souligné avec insistance et de manière convaincante que «l'image » représentait un nouveau paradigme qu'il importait de prendre en considération sur le plan théorique.

5 Boehm et Mitchell ne sont pas du tout les seuls protagonistes théoriques de ce nouveau paradigme de l'image. Dans l'espace germanophone, ce sont surtout Hans Belting et Horst Bredekamp qui ont, chacun à sa manière, tenté de libérer la question de l'image de son enfermement dans l'histoire de l'art. Tandis que, dans un grand nombre de publications, Hans Belting cherche à transposer l'histoire de l'art en une science de l'image, profilant la question de l'image comme une question anthropologique fondamentale et projetant ainsi une science de l'image comme anthropologie de l'image ${ }^{13}$, Horst Bredekamp entreprend, quant à lui, la tentative de lier science de l'image et science de l'art, d'imbriquer des questionnements relevant de la science de l'image et des concepts et méthodes venant de la science de l'art, autrement dit de comprendre la science de l'image comme une recherche sur l'image. ${ }^{14} \mathrm{Ce}$ qui intéresse Bredekamp est une nouvelle réflexion sur l'image d'orientation pragmatique, au sens philosophique du terme, une théorie de l'acte imagé qui serait également constitutif pour la pensée, et qui inclurait du coup une dimension politique et sociale : les images sont formatrices [prägend] d'histoire, d'agir et de théorie. «Un acte imagé engendre des faits, en ce qu'il met des images dans le monde», est-il annoncé dans son essai «Bildakte als Zeugnis und Urteil » [Des actes imagés comme témoignage et jugement], qui a été choisi pour cette première édition de la revue Trivium. Beaucoup d'autres noms seraient à citer : par exemple Gerhard Paul, qui tente de faire avancer en Allemagne le projet d'une "Visual History ", Jörg R. J. Schirra, qui travaille plus particulièrement dans le domaine de l'informatique visuelle, ou bien Klaus Sachs-Hombach, le protagoniste de la science de l'image.

6 "Sciences de l'image » évoque aussi le nom de cette nouvelle discipline qui englobe toutes les disciplines scientifiques traditionnelles qui « contribuent à la compréhension théorique de la thématique de l'image $»^{15}$. Ainsi le formule Klaus Sachs-Hombach, lequel, en tant qu'éditeur de plusieurs volumes sur le sujet, tente de conférer un profil théorique à la science de l'image. Le spectre ainsi couvert va des sciences sociales et de la nature aux sciences des médias, en passant par les disciplines historiques et appliquées.

7 C'est également au niveau institutionnel que s'est établie, au cours des dernières années, une réflexion interdisciplinaire sur les images et la représentativité - et ce bien au-delà des frontières des domaines classiques de la recherche sur l'image que sont la science de l'art et l'archéologie. Des premiers cursus d'étude ont même été fondés autour de la science de l'image. Il faut par exemple mentionner Eikones: le plus important projet de recherche national suisse intitulé "Bildkritik. Macht und Bedeutung der Bilder [Critique de l'image. Puissance et signification des images]», dirigé par Gottfried Boehm; le programme de recherches doctoral «Bild-KörperMedium [Image-corps-médium] », dont le premier porte-parole, Hans Belting, a depuis 
été remplacé dans cette fonction par Beat Wyss ; le site Internet du projet de recherche «Iconic Turn » de la fondation Hubert Burda ; le département des sciences de l'image de l'Université du Danube à Krems ; l'institut virtuel des sciences de l'image ; ou encore la revue pour une recherche interdisciplinaire en science de l'image, pour n'en citer qu'un petit nombre. On trouvera d'autres informations sur la liste de liens explicitement commentée.

8 Le choix des contributions pour cette édition de Trivium tente, en dépit des limites imposées, de satisfaire, à travers huit textes en tout, à l'ambition de présenter un débat et - c'est notre espoir - d'inciter à le poursuivre. Si Hans Belting n'est représenté par aucun texte, c'est uniquement parce qu'un grand nombre de ses livres et essais sont déjà disponibles en traduction française. Nous avons retenu en revanche un texte programmatique de Gottfried Boehm, qui se trouve aussi bien dans son volume Iconic Turn - qui a entraîné de très importants effets sur le débat en Allemagne -, que dans son dernier et important livre Wie Bilder Sinn erzeugen [Comment les images engendrent $\mathrm{du}$ sens]. La contribution retenue de Lambert Wiesing est exemplaire pour une nouvelle réflexion philosophique sur la représentativité, et le texte de Sigrid Weigel, "Les Images, acteurs majeurs de notre pensée. À propos de la poiesis et de l'episteme des images langagières et visuelles » dresse un état des lieux à la fois sobre et saturé de théorie des analyses théoriques du déluge des images, qui, bien que souvent conjurées, très critiquées et déplorées, rappellent l'Iconic Turn à sa tâche : être aussi une réflexion sociale.

Étant donné qu'au cours de ces dernières années de nombreux instituts, blogs, centres de recherche, revues etc. se sont résolument tournés vers des questions relevant de la science de l'image, nous avons également établi, à côté d'une bibliographie sélective relativement resserrée, un répertoire aussi bien allemand que français d'adresses Internet. Notre espoir est que ces liens deviennent aussi les liens d'un débat francoallemand, qui, à bien des égards, reste encore à venir.

\section{BIBLIOGRAPHIE}

Belting, Hans, Das Ende der Kunstgeschichte?, Munich, 1983.

Belting, Hans, Das Ende der Kunstgeschichte. Eine Revision nach 10 Jahren, Munich, 1995.

Belting, Hans, Image et culte. Une histoire de l'art avant l'époque de l'art, trad. de l'allemand par Frank Muller, Les éditions du Cerf, Paris, 1998.

Belting, Hans, Pour une anthropologie des images, trad. de l'allemand par Jean Torrent, 346 p., ill. en noir et ill. en coul, Paris, Gallimard, 2004 [coll. Le temps des images].

Belting, Hans (éd.), Bilderfragen. Die Bildwissenschaften im Aufbruch, Munich, 2007.

Boehm, Gottfried (éd.), Was ist ein Bild? Munich, 1994, ${ }^{2} 1995$.

Boehm, Gottfried, Wie Bilder Sinn erzeugen. Die Macht des Zeigens, Berlin, 2007. 
Bredekamp, Horst, Kunst als Medium sozialer Konflikte. Bilderkämpfe von der Spätantike bis zur Hussitenrevolution, Francfort/Main, 1975.

Bredekamp, Horst, Antikensehnsucht und Maschinenglauben. Die Geschichte der Kunstkammer und die Zukunft der Kunstgeschichte, Berlin, 1993.

Bredekamp, Horst, « A Neglected Tradition? Art History as Bildwissenschaft », in : Critical Inquiry, vol. 29, 2003, p. 418-428.

Bredekamp, Horst et. al. (éd.), Bildwelten des Wissens, Berlin, 2003.

Bredekamp, Horst, Bilder bewegen. Von der Kunstkammer zum Endspiel, Berlin, 2007.

Breidbach, Olaf, Bilder des Wissens. Zur Kulturgeschichte der wissenschaftlichen Wahrnehmung, Munich, 2005.

Dikovitskaya, Margarita, From Art History to Visual Culture: The Study of the Visual after the "Cultural Turn », Cambridge/Mass., 2005.

Frank, Gustav, « Textparadigma kontra visueller Imperativ: 20 Jahre Visual Culture Studies als Herausforderung der Literaturwissenschaft ", in : Internationales Archiv für Sozialgeschichte der deutschen Literatur, vol. 31, cahier 2, 2006, p. 26-89.

Imdahl, Max, Gesammelte Schriften, 3 vol, éd. par Gottfried Boehm et. al., Francfort/Main, 1996.

Jay, Martin, Downcast Eyes. The Denigration of Vision in the Twentieth Century Thought, Berkeley, 1993.

Kemp, Martin, Bilderwissen. Die Anschaulichkeit naturwissenschaftlicher Phänomene, Cologne, 2003.

Maar, Christa / Burda, Hubert (éd.), Iconic Turn. Die neue Macht der Bilder, Cologne, 2004.

Maar, Christa / Burda, Hubert (éd.), Iconic Worlds. Neue Bilderwelten, Cologne, 2006.

Mirzoeff, Nicholas (éd.), The Visual Culture Reader, Londres / New York, 1998.

Mirzoeff, Nicholas, An Introduction to Visual Culture, Londres / New York, 1999.

Mitchell, W. J. T., Picture Theory. Essays on verbal and visual representation, Chicago, 1994.

Mitchell, W. J. T., What do pictures want?, Chicago, 2005.

October: «Questionnaire on Visual Culture », in : October, Nr. 77, 1996, p. 3-4 et 25-70.

Paul, Gerhard (éd.), Visual History. Ein Studienbuch, Göttingen, 2006.

Sachs-Hombach, Klaus, Das Bild als kommunikatives Medium. Elemente einer allgemeinen Bildwissenschaft, Cologne, 2004.

Sachs-Hombach, Klaus (éd.), Wege zur Bildwissenschaft. Interviews, Cologne, 2004.

Sachs-Hombach, Klaus (éd.), Bildwissenschaften. Disziplinen, Themen, Methoden, Francfort/Main, 2005.

Schulz, Martin, Ordnungen der Bilder. Eine Einführung in die Bildwissenschaft, Munich, 2005.

Warburg, Aby, Gesammelte Schriften, éd. par Horst Bredekamp et. al., Berlin, 1998 sq.

Wiesing, Lambert, Artifizielle Präsenz. Studien zur Philosophie des Bildes, Francfort/Main, 2005. 


\section{NOTES}

1. Toutefois, Rorty recourt, ce faisant, à l'essai de Gustav Bergmann, «Two Types of Linguistic Philosophy ", in : id., The Metaphysics of Logical Positivism, New York, 1954, p. 106-131, qui utilise déjà ce concept. Voir également sur ce point Mike Sandbothe, "Die pragmatische Wende des linguistic turn ", in : id. (éd.), Die Renaissance des Pragmatismus, Weilerwist, 2000, p. 96-126.

2. Richard Rorty, "Introduction », in : id. (éd.), The Linguistic Turn. Recent Essays in Philosophical Method, Chicago, 1967, p. 1-41, p. 39.

[N.d.T. : « Une fois surmontée la conception "spectatrice" de la connaissance, la conception qui la remplacera conduira à de nouvelles formulations partout ailleurs en philosophie, et tout particulièrement dans la méta-philosophie. »].

3. Richard Rorty, L'Homme spéculaire, trad. de l'anglais par Thierry Marchaisse, Seuil, Paris, 1990 [coll. L'Ordre philosophique].

4. Ibid., p. 22.

5. Sous une forme ramassée, ils résument dans deux lettres comment ils en sont venus à cette formation de concept et quels programmes théoriques - tout à fait divergents - y étaient liés : Gottfried Boehm, «Iconic Turn, Ein Brief [Une lettre]»; W. J. T. Mitchell, «Pictorial Turn. Eine Antwort [Une réponse]», in: Belting, Hans (éd.), Bilderfragen. Die Bildwissenschaften im Aufbruch, Munich, 2007, p. 27-47.

6. Boehm, in : Belting (2007), p. 30.

7. Voici comment Horst Bredekamp résume les conséquences de l'iconic turn deux décennies plus tard: «Si l'archéologie et l'histoire de l'art, qui sont les disciplines originelles de l'image, ont réactivé leurs traditions en tant que sciences historiques de l'image, si la science du film place au premier plan, avant le récit, le caractère imagé des films, si la philosophie privilégie la réflexion sur l'image, si les sciences littéraires analysent la relation réciproque entre écriture et image, si l'historiographie libère les sources de l'image du sceau infamant de l'illustration, si l'histoire des sciences souligne le conditionnement visuel de la Science, si la jurisprudence travaille à une iconologie du droit, si dans les mathématiques la formule "Seeing is believing", dirigée contre l'iconoclasme du groupe Bourbaki, continue à produire ses effets, si la biologie, pour la première fois depuis Darwin, évoque le critère de la beauté dans la sélection naturelle, et si le visuel informatique agissant dans tous les champs des sciences de la nature est analysé, alors ce sont là les signes que, dans le champ de la recherche également, a lieu une transformation profonde appelée par les techniques modernes de l'image et par le désir d'une participation visuelle, transformation qui s'accomplit dans l'ensemble de la culture." Horst Bredekemp, «Drehmomente - Merkmale und Ansprüche des iconic turn», in : Maar, Christa / Burda, Hubert (éd.), Iconic Turn. Die neue Macht der Bilder, Cologne, 2004, p. 15-26, p. 17.

8. Et plus explicitement, deux ans plus tard, dans son livre Picture Theory, Chicago, 1994.

9. Voir Mitchell, in : Belting (2007), p. 43.

10. Willibald Sauerländer, Iconic Turn? Eine Bitte um Ikonoklasmus, in: Maar / Burda (2004), p. 407-426, p. 411.

11. Gottfried Boehm (éd.), Was ist ein Bild?, Munich, 1994.

12. Boehm, in: Belting (2007), p. 29. Voir également le recueil d'essais programmatiques: Wie Bilder Sinn erzeugen. Die Macht des Zeigens [Comment les images engendrent du sens. La puissance de la monstration], Berlin, 2007.

13. Voir par ex. Hans Belting, Pour une Anthropologie des images, trad. de l'allemand par Jean Torrent, Paris, Gallimard, 2004 [coll. Le temps des images].

14. Voir par ex. l'entretien avec Hans Belting : «Im Königsbett der Kunstgeschichte », in : Die Zeit, 6 avril 2005. 
15. Klaus Sachs-Hombach, «Konzeptionnelle Rahmenbedingungen zur interdisziplinären Bildwissenschaft », in : id., Bildwissenschaften. Disziplinen, Themen, Methoden, Francfort/Main, 2005, p. 11-20, p. 13.

INDEX

Schlüsselwörter : Bildtheorie, Iconic turn, Kunstgeschichte, Linguistic turn Mots-clés : histoire de l'art, iconic turn, linguistic turn, théorie de l'image

\section{AUTEURS}

\section{BERND STIEGLER}

Professeur de littérature, Université de Constance 